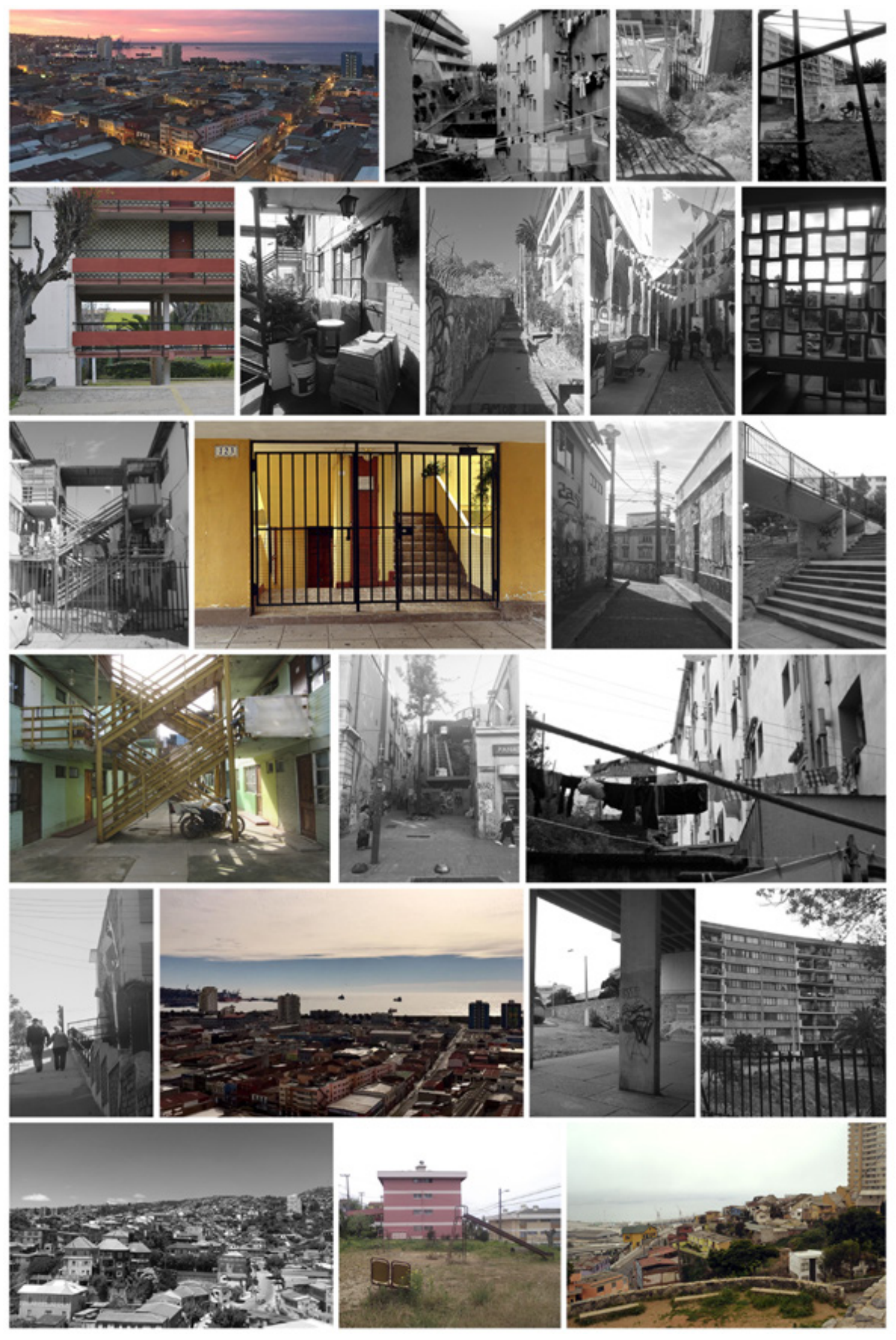



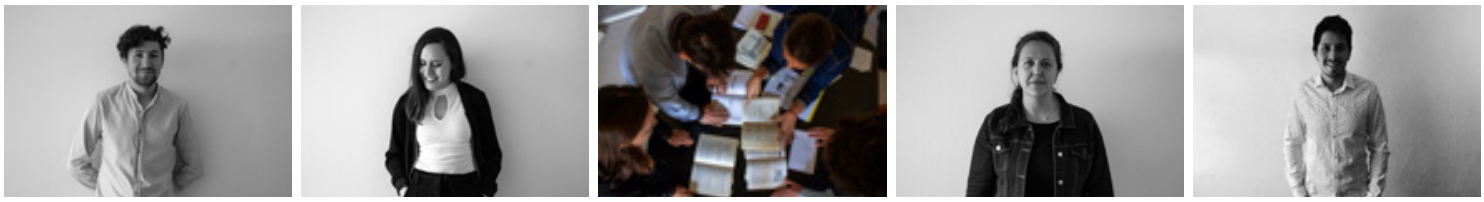

Secuencia: Universidad Técnica Federico Santa María, Valparaíso, Chile Fotos: Fotografías por los autores

\title{
USO, DESUSO Y LA CARACTERIZACIÓN DE LA DIMENSIÓN INTERMEDIA COMO UN POTENCIAL DE REHABILITACIÓN: TRES ESCALAS DE APROXIMACIÓN, ANÁLISIS Y DIAGNÓSTICO PARA EL CASO DE VALPARAÍSO, CHILE'
}

\author{
USE, DISUSE AND THE CHARACTERIZATION OF THE INBETWEEN DIMENSION AS \\ A POTENTIAL AREA FOR REHABILITATION: THREE SCALES OF APPROXIMATION, \\ ANALYSIS AND DIAGNOSIS FOR THE CASE OF VALPARAÍSO, CHILE'
}

Carolina Carrasco Walburg ${ }^{2}$, Christian Alvial Araya ${ }^{3}$, Nicole Eujenio ${ }^{4}$, Sebastián Rojas 5

RESUMEN

El transcurrir de la vida de los edificios y el fenómeno de obsolescencia son procesos claramente apreciables en el caso de Valparaíso. Dadas las características morfológicas y los modos de vida asociados, se presume que cualquier actuación de rehabilitación ha de asumir un enfoque integral abarcando las dimensiones material, funcional, social y ambiental. Para el presente trabajo el cómo mejorar ese deterioro es materia de investigación exploratoria, con el objetivo de identificar los focos de problemas y oportunidades para la rehabilitación arquitectónica sostenible. Se introducen tres potenciales de actuación, en este contexto, manifiestos en la 'dimensión intermedia', que permiten planificar una intervención: el potencial de confort, la capacidad de autoregeneración socio-espacial y la oportunidad de revitalización; detectados mediante una metodología cualitativa de análisis arquitectónico. Con diferentes instrumentos de reconstitución socioespacial y apoyo teórico, se elaboran diagnósticos de obsolescencia en las preexistencias que conducen a la formulación de nuevas actuaciones.

Palabras clave: rehabilitación, territorio, escalas, análisis, desarrollo sostenible.

ABSTRACT

The life of buildings over time and the phenomenon of obsolescence are clearly evident processes in the case of Valparaíso, Chile. Given the morphological characteristics and the associated ways of life, it is presumed that any rehabilitation action must take on a comprehensive approach by covering the material, functional, social and environmental dimensions. For the present study, exploratory research on how to improve this deterioration was carried out with the objective of developing a multiscale tool for characterizing problems and opportunities for sustainable architectural rehabilitation. Using a qualitative architectural analysis methodology, three potential areas for action were identified. Present in the "intermediate dimension," they make it possible to plan an intervention and include: the potential for comfort, the ability for socio-spatial self-regeneration, and the opportunity for revitalization. With different socio-spatial reconstruction instruments and theoretical support, obsolescence diagnostics were prepared taking into consideration the pre-existing conditions, which lead to the formulation of new interventions.

Keywords: rehabilitation, territory, scales, analysis, sustainable development.

Artículo recibido el 6 de agosto de 2018 y aceptado el 18 de diciembre de 2018 DOI: https://doi.org/10.22320/07196466.2018.36.054

[1] Esta investigación se enmarca en el programa de magíster Rehabilitación Arquitectónica Sostenible (MRA) impartido por el Departamento de Arquitectura de la Universidad Técnica Federico Santa María desde el año 2017.

[2] Departamento de Arquitectura, Universidad Técnica Federico Santa María, Valparaíso, Chile. carolinacarrascowalburg@gmail.com

[3] Departamento de Arquitectura, Universidad Técnica Federico Santa María, Valparaíso, Chile. christian.alvial@gmail.com

[4] Departamento de Arquitectura, Universidad Técnica Federico Santa María, Valparaíso, Chile. nicole.eujenio@gmail.com

[5] Departamento de Arquitectura, Universidad Técnica Federico Santa María,Valparaíso, Chile.sebastian.rojasve@alumnos.usm.cl 
Valparaíso es una ciudad morfológicamente compleja dada la conformación de sus cerros, quebradas y estrechez de planicie, por ello se ha adaptado a las condiciones territoriales. Las singularidades arquitectónicas se deben principalmente a la estrechez espacial, la oblicuidad, la irregularidad de la trama urbana y la relación con el paisaje (Figura 1), donde la vista y el asoleamiento se constituyen como bienes colectivos. La relación entre bordes y pliegues, en un contexto de entrelazamientos difusos entre lo público y lo privado, moldeó un hábitat entre interiores y exteriores, influidos por el clima, determinando respuestas arquitectónicas y modos de habitar propios de la ciudad (Carrasco, 2015: 65).

No obstante, desde años se ha ido incrementando el deterioro del hábitat construido, afectandoalosedificios, espacios urbanos y al entrelazamiento entre ambos. Según Goldberger, hay tres hechos que determinan estas transformaciones: los inmuebles cambian porque nosotros cambiamos y esto se plasma en las actitudes que tenemos hacia la arquitectura; los edificios en sí también cambian, se amplían, se maquillan, cambian los usuarios, se vuelven más familiares, etc.; y, por último, en la medida que la cultura se modifica, igualmente lo hace el modo en que vemos los edificios (2012: 206-207). Calduch (2009), por su parte, indica que la obsolescencia surge cuando los requerimientos del momento actual no se adaptan a la función originaria de una preexistencia ni al espacio asociado. Esta problemática no solo ocurre en lo funcional sino que puede ampliarse 

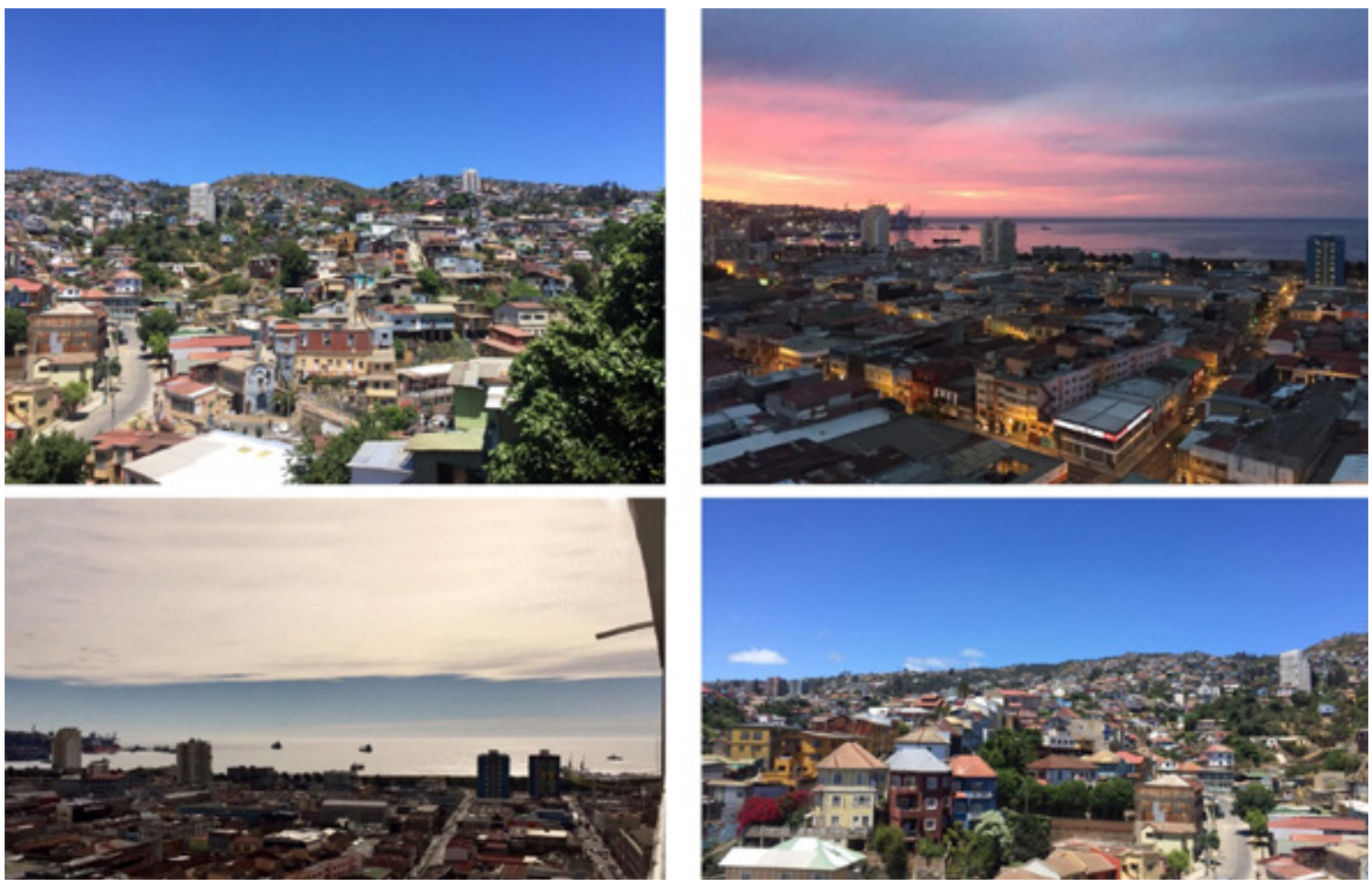

Figura 1 Vista generalizada de Valparaíso. Fuente: Fotografías de Carolina Carrasco, 2018.

a los materiales, la gestión y la pérdida de significado cultural, lo que se observa en muchas edificaciones y espacios urbanos de la ciudad, y que no ha logrado ser aplacado por las acciones implementadas tras la inscripción del casco histórico como Patrimonio de la Humanidad de la UNESCO (2003).

Si los atributos de la ciudad efectivamente tienen que ver con varias escalas entrelazadas por la relación entre arquitectura, paisaje, lo común y la accesibilidad, ¿cómo rehabilitar adecuada y sosteniblemente en una ciudad como esta? Como el fortalecimiento del espesor limítrofe entre territorios es la condición que favorece la vida de barrio y la interrelación entre la arquitectura, la ciudad y el medio en que se inserta, entonces se observa una oportunidad de actuación presente en el territorio de lo intermedio, en cuanto a integrar o aislar el mundo interior con el exterior mediante una acción. Por ello, se infiere que la rehabilitación sostenible requiere estar vinculada a la revitalización, como también hacerse cargo de este territorio que no solo compete a la arquitectura, sino que ha de incluir a la comunidad vinculada al inmueble y al contexto, para que se sostenga en el tiempo. Pero, ¿qué se entiende por "lo intermedio"? Variados autores, como Van Eyck, Hertzberger, Norberg-Schulz, entre otros, se han referido a este concepto. Glenda Kapstein presenta una visión específica en relación al clima y al territorio, donde lo intermedio corresponde a un espacio de transición y existe una ligazón directa con la realidad física del exterior -paisaje y clima-, que da paso a la composición espacial del espacio intermedio, es decir, un mediador entre un ambiente interno controlado y otro externo no controlado (2015: 62). Ahora bien, en el trabajo aquí expuesto la "dimensión intermedia" no solo abarca al espacio o la relación entre la casa y la ciudad (Van Eyck, 1956: 133), además incluye lo experiencial y la formación de lugar (Norberg-Schulz, 1975: 31). Esta condición de lugar resulta relevante cuando paisaje y geografía determinan los modos de vida (Tuan, 1977), ya que ello genera la "domesticación" del espacio, es decir, la construcción, la transformación y el sometimiento a reglas de uso (Bailly, 1989: 15). El lugar suele configurarse en una condición intermedia e impulsa la consolidación del hábitat construido. Es por esto que arquitectura y lugar son conceptos inseparables relacionados por el espacio intermedio que, a su vez, definen la idea de lugar y construyen ciudad (Kapstein, 2015: 62). Entonces, se concibe la "dimensión intermedia" como el territorio -tiene medidas, elementos y relaciones topológicasdonde se inscriben las dualidades del espacio y los pares opuestos (Van Eyck, 1962: 602), es decir, lo material y lo inmaterial, interior y exterior, abierto y cerrado, permanencia y movimiento, lo propio y lo colectivo, etc. En este territorio ocurre el acto del encuentro de los individuos en una recíproca presencia (Buber, 1967: 147). Para indagar en las formas rehabilitación a partir de esta dimensión intermedia, se plantea como objetivo de la investigación identificar los focos presentes en este territorio, que permitan la formulación de la rehabilitación arquitectónica sostenible. 


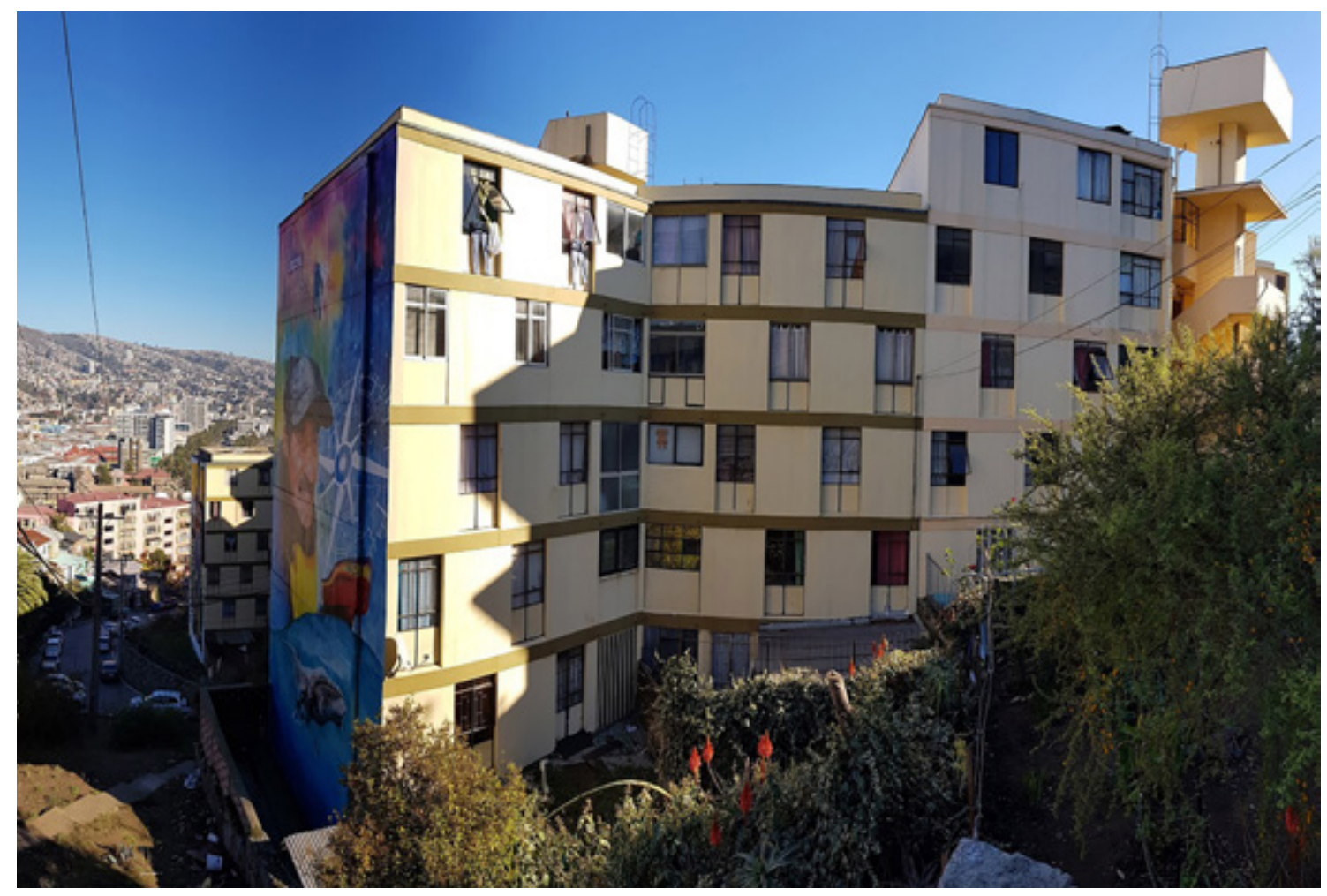

Figura 2 Vista de la Población José Ignacio Zenteno de Valparaíso. Fuente: Fotografía de Sebastián Rojas, 2018.

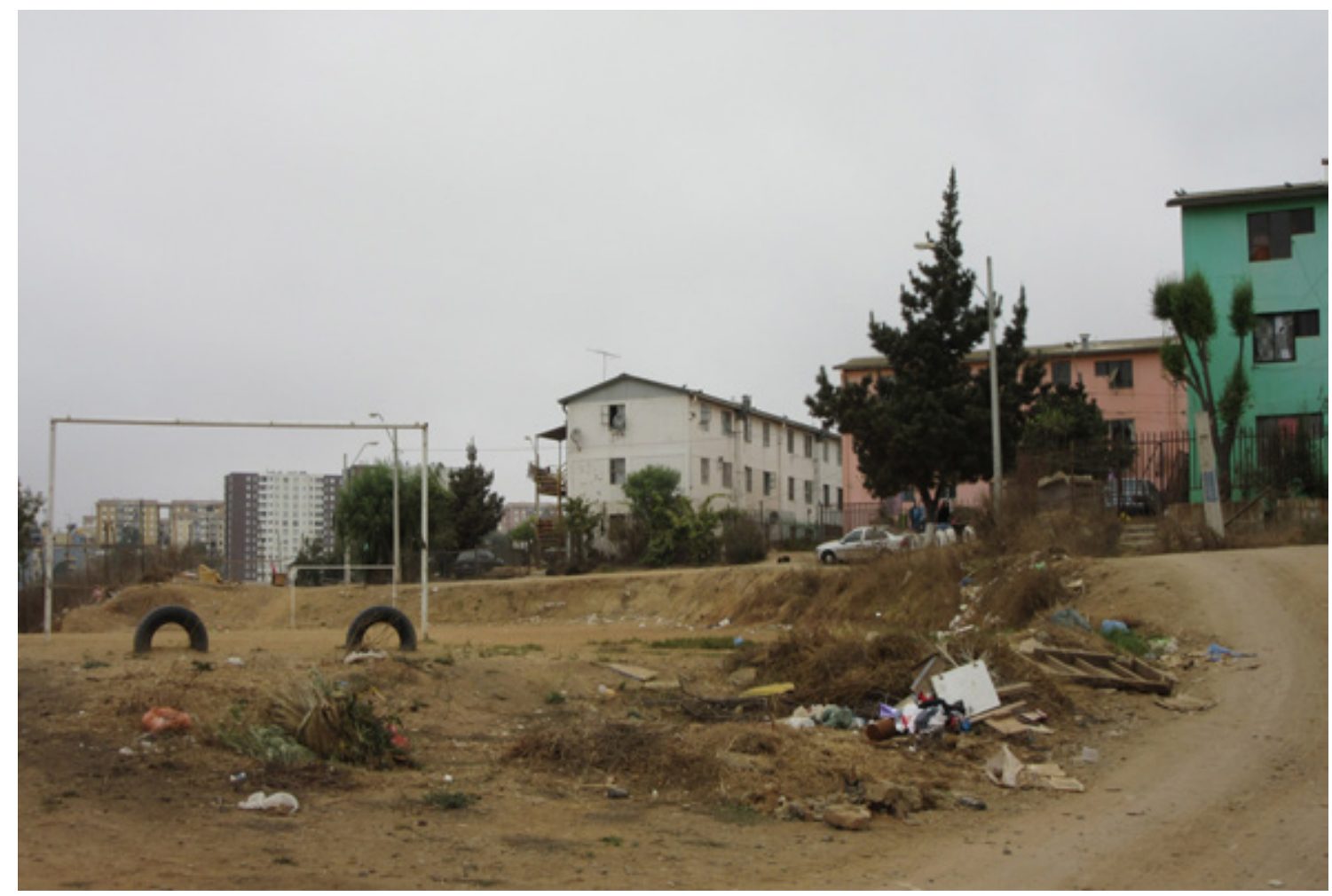

Figura 3 Cancha de fútbol en avanzado grado de obsolescencia. Población "Glorias Navales", Viña del Mar. Fuente: Fotografía de Christian Alvial, 2018. 


\section{MÉTODO}

La oportunidad de intervenir esta área donde confluyen diferentes órdenes es generando articulaciones espacialesparafacilitarelencuentroyasíregularladivisión y tensión generada entre territorios con diferentes demandas (Hertzberger, 1991: 51). Pero, ¿cómo abordar esta articulación en la rehabilitación y, específicamente, en el caso del gran Valparaíso? Para abarcar la totalidad se decidió caracterizar esta problemática desde tres escalas, donde cada una reconoce el modo particular de esta dimensión intermedia y los problemas asociados. Este criterio se basó en el planteamiento teórico desarrollado por Calzada, Chautón y Di Siena (2013), para quienes las oportunidades de innovación están presentes en estas tres escalas con diversas características y potencialidades. Así, se procedió a definir la microescala del escenario doméstico y el edificio, la escala meso de lo comunitario y las agrupaciones, y la macroescala de lo urbano. Se abordó una metodología cualitativa de carácter exploratorio que integró la teorización, el análisis de casos, la observación directa y, en algunos casos, la percepción de los habitantes. Entonces, se elaboró un marco teórico para cada enfoque, que permitió identificar los parámetros asociados y se formularon tres herramientas respectivas de levantamiento de las condiciones de un caso de estudio genérico, que fundamentaron el diseño de una matriz de análisis de la dimensión intermedia, el estado de deterioro del caso y las oportunidades para la rehabilitación. Esta herramienta fue probada en un caso específico, lo que facilitó el ajuste de los parámetros.

En la microescala, se identificó y caracterizó la dimensión intermedia a partir de las cualidades del edificio y la vivienda, además de la percepción y el significado que el habitante tiene sobre los límites de la vivienda y su relación con el entorno. Primero, se trabajó en la descomposición de las características primitivas del edificio en relación a su influencia sobre las variables de confort establecidas, tomando como punto de partida la categorización propuesta en el libro Climate Design (Hausladen et al., 2005), que posteriormente acuña Elena Lucchi (2015), presentando una aproximación a través de los sentidos del cuerpo humano que definen el confort ambiental desde la visión, la audición, la sensación y el olfato. Complementariamente, se usó el instrumento de entrevistas semi-estructuradas donde se fijaron algunas preguntas estratégicas como, por ejemplo, ¿cómo considera la iluminación natural de los espacios comunes de su edificio?, y otras más generales, como ¿cuál es su percepción con respecto a la visión? Además, para aplicar esta herramienta se identificó un caso de estudio motor, el Conjunto habitacional José
Ignacio Zenteno de Oscar Zaccarelli V., ubicado en el cerro Los Lecheros de Valparaíso (Figura 2) y construido como parte del Plan Habitacional de la CORVI entre 1961 y 1964. La selección del caso corresponde a las condiciones de obsolescencia y a la presencia de un grupo de 10 habitantes que accedieron a colaborar con la investigación. Esta situación es fundamental ya que permite indagar en la relación entre habitante y suelo, la preferencia por las vistas predominantes, las prácticas al interior de la vivienda, los parámetros de asoleamiento, el uso y desuso del espacio común, entre otros factores, para luego tomarlos como punto de partida en las estrategias de intervención.

En la meso escala, se realiza un análisis a través de un caso motor, la Población Glorias Navales (Figura 3), como herramienta de aproximación a la realidad del "hábitat construido". Para ello, se identificaron cuatro dimensiones claves que estructuran una matriz de análisis: ciudad, soporte físico, sociedad y sostenibilidad; basadas en investigaciones previas teórico/prácticas (Chacón, 2012; Montaner, Muxí y Falagán, 2011), pero adaptadas a la realidad local, y complementadas por un "sociograma" 6 que representa la capacidad de actuación socioespacial presente. Este sociograma recoge la información cualitativa obtenida de entrevistas con informantes claves ${ }^{7}$, con quienes se establece un nexo de confianza mayor debido a su fuerte vinculación con el territorio como interventores ${ }^{8}$. Ellos ya han decantado esta información y conocen las necesidades a considerar en la rehabilitación. También se conversó con informantes informales ${ }^{9}$, a quienes se les formularon preguntas específicas sobre su vinculación al territorio. Se aplicaron 15 encuestas $^{10}$ a usuarios informales que tienen relación indirecta con la comunidad, ya sea por manejar negocios locales o hacer la mantención de huertos urbanos y áreas comunes. Las preguntas se enfocaron en determinar los principales lugares de encuentro entre vecinos, las áreas de dispersión más utilizadas, la participación en actividades comunitarias y las mejoras espaciales posibles $y$ anheladas.

En la macroescala, se planteó un sistema de análisis que evalúa la revitalización del entorno del edificio preexistente ${ }^{11}$, basada en conceptos teórico/prácticos de investigaciones previas (Bentley et al., 1999; Gehl, 2006; Montaner, Muxí y Falagán, 2011) e incorporando la realidad de Valparaíso. Este análisis consistió en la toma de datos en terreno mediante una ficha que compila los elementos de revitalización presentes en tres dimensiones relevantes: la dimensión del edificio, su entorno cercano y el barrio donde se encuentra; para luego procesar la información a través de la valoración de la revitalización. Se abordó la preexistencia como encrucijada de la complejidad actual en la que

[6] Representación gráfica de la red de personas o entidades conectadas al territorio específico estudiado.

[7] Informantes claves: aquellos que tienen relación directa con el lugar, ya sea por ser habitante y/o representar al colectivo generando cierto grado de control sobre la gestión y acción del territorio.

[8] Interventores: aquellos que sin ser habitantes actúan o gestionan el territorio

[9] Informantes informales: Habitantes recurrentes que en alguna medida han establecido un vínculo a algún sector del territorio participando activamente en él.

[10] Cabe destacar que las entrevistas se hicieron en 2 meses de trabajo (octubre-noviembre) y la participación fue mayor con las mujeres que habían encauzado sus anhelos por mejorar las condiciones de vida de su familia y vecinos, expresando así una faceta de liderazgo dentro de la comunidad.

[11] Se trata, específicamente, de edificios que ya han sido rehabilitados en Valparaíso y que poseen actividades que aportan al entorno y el encuentro entre personas. 


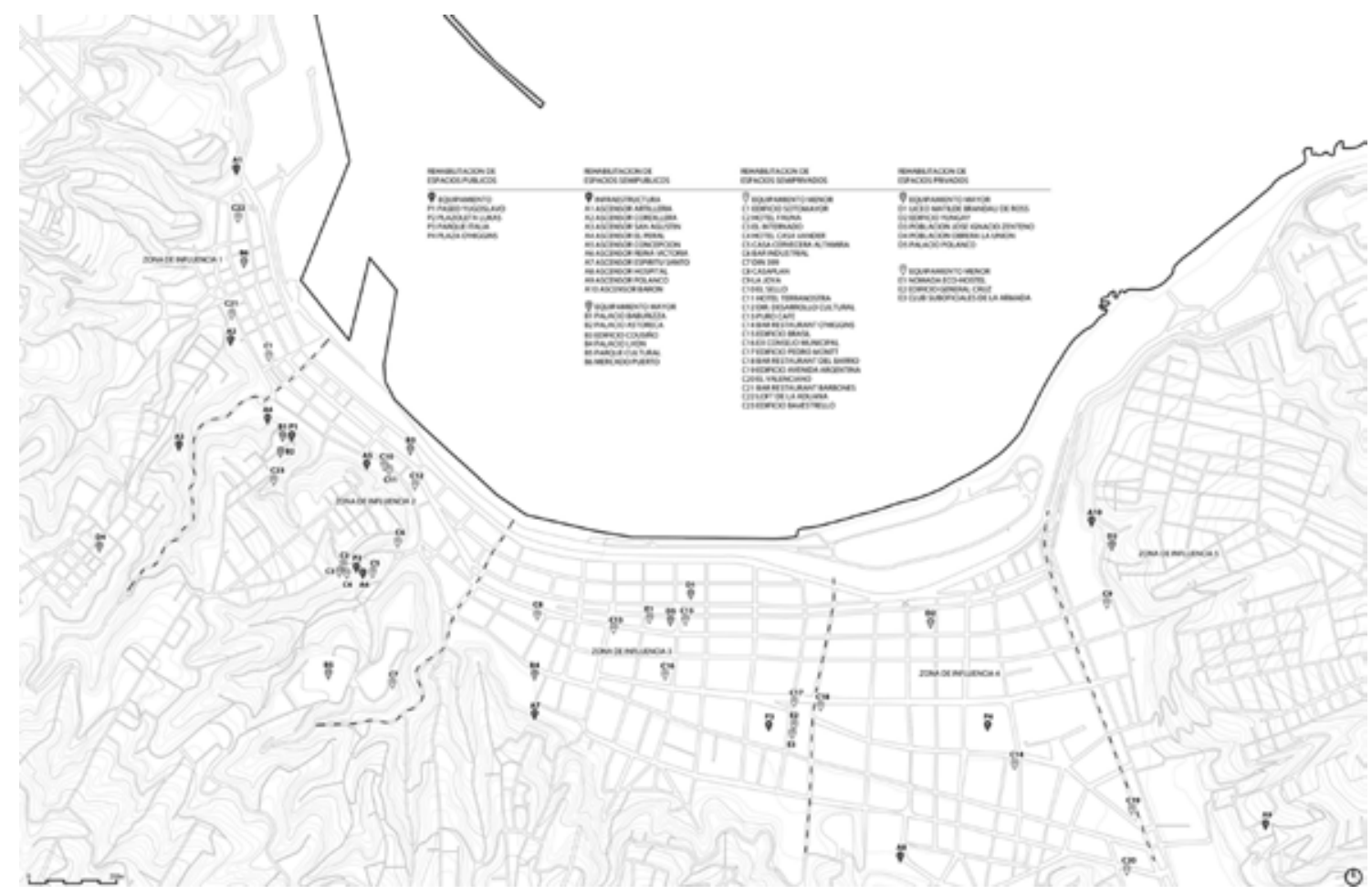

Figura 4 Ubicación de la primera aproximación a la selección de los casos de estudio. Fuente: Elaboración de Nicole Eujenio.

convergen, a través de la arquitectura, cuestiones urbanas y sociales. En este caso, los límites entre los espacios privados del edificio y el espacio público -la dimensión intermedia-, se vuelve el objeto relevante a la hora de la rehabilitación. Este espacio debe plantearse de forma tal que permita la coexistencia de una variedad de actividades interiores privadas con la naturaleza de las actividades del espacio público (Bentley et al., 1999).

En cuanto a la selección de casos, la primera aproximación cuenta con la identificación de 51 obras de rehabilitación ejecutadas desde el 2007 hasta la fecha, en las que se encuentran espacios públicos, semipúblicos, semiprivados y privados (Figura 4), que ayudan a comprender las relaciones y efectos en cadena que las actuaciones de rehabilitación producen en el sistema urbano. Mientras que, para la evaluación de la revitalización, se seleccionan los edificios intermedios entre lo público y lo privado, quedando una muestra de 39 casos para la comparación de las rehabilitaciones. Finalmente, los 23 casos de edificios semiprivados se ordenaron en 3 familias $^{12}$, seleccionando un ejemplar de cada una para el análisis de las relaciones entre la revitalización y la rehabilitación en la dimensión intermedia (Tabla 1).
Tras la aplicación de las herramientas de análisis a los distintos casos de estudio, se advirtió que en todas las escalas la dimensión intermedia tiene sus propias cualidades y conlleva un foco o potencial de actuación que promueve una estrategia de rehabilitación arquitectónica sostenible. Por lo tanto, como producto final se presentan estos tres potenciales de actuación y sus respectivas oportunidades.

\section{RESULTADOS}

\section{Microescala: El potencial de confort}

En la dimensión doméstica, el uso y desuso surge como consecuencia de la incompatibilidad entre las características primitivas de la edificación y las actuales demandas del estilo de vida, lo que conlleva ineludiblemente al deterioro de las condiciones de confort ambiental de sus habitantes. Cuando se trata de la vivienda colectiva dicha incompatibilidad se refleja en el límite, el borde, el espacio intermedio, la envolvente y las estancias. En este sentido, la herramienta de 


\begin{tabular}{|c|c|c|c|}
\hline \multirow{2}{*}{ CATEGORIAS } & \multicolumn{3}{|l|}{ SELECCION CASOS DE ESTUDIO } \\
\hline & PRIMERA ETAPA & SEGUNDA ETAPA & TERCERA ETAPA \\
\hline \multirow{4}{*}{$\begin{array}{l}\text { Espadios } \\
\text { publicos }\end{array}$} & P1 Pareo Yugoslavo & & \\
\hline & P2 Plasoleta tukas & & \\
\hline & P3 Parque ltala & & \\
\hline & P4 Plaza OHiggins" & & \\
\hline \multirow{16}{*}{$\begin{array}{l}\text { Espadios } \\
\text { semelpublicos }\end{array}$} & Al Ascenser Aatilleris & A1 Ascensor Antileria & \\
\hline & A2 Ascensor Condillers" & A2 Ascensor Cordillera" & \\
\hline & A3 Ascensor San Agustin & A3 Ascensor San Agustin & \\
\hline & A4 Ascenser B Peral & A4 Ascensor E Peral & \\
\hline & As Ascensor Concepción" & As Ascensor Concepcion" & \\
\hline & As Ascensor Reina Victoria & As Ascensor Reina Victeria & \\
\hline & A7 Ascenser Espintu Santo & A7 Ascensor Espiritu Santo & \\
\hline & As Ascensor Hospital Van Buen & As Ascensor Hospital Van Buren & \\
\hline & A9 Ascensof Polanco & A9 Ascensor Polanco & \\
\hline & Alo Ascensor banba & A10 Accensor Rarch & \\
\hline & B1 Palacio Raburizza & B1 Palacio Baburizza & \\
\hline & B2 Palacio Astoreca & B2 palacio Astoreca & \\
\hline & B3 Edifikio Cousith & B3 Edficio Cousilo & \\
\hline & BA palacio tyon & BA palakio tyon & \\
\hline & BS Parque Cultural & BS Parque Cultural & \\
\hline & 86. Mercado Puento* & B6 Mertado Puerso" & \\
\hline \multirow{23}{*}{$\begin{array}{l}\text { Espadios } \\
\text { semipeivados }\end{array}$} & C1 Edificio Sotomuyor & C1 Edificio Sotomayor & C1 Edificio Sotomayor \\
\hline & C2 Hotel Fauna & C2 Hotel Fanu & C2 Hotel Faun \\
\hline & C3 Elingernaso & C3El intemado & CBEl Internado \\
\hline & C4 Hotel Casa Vander & C4 Hotel Cosa Viander & C4 Hotel Cona Vander \\
\hline & C5 Cra Cemoera Ntamira & C5 Casa Cervecera Altamin & Cs Casa Cenvecera Altamia \\
\hline & C6 Rar industral & C6 Rar hodustrial & C6 Bar industrial \\
\hline & $\cos 399$ & $0 \operatorname{Din} 399$ & 00 in 399 \\
\hline & C8crapian & c8casabian & C8casaplan \\
\hline & C9laboy & C9La bya & C9La doya \\
\hline & C10 El sello & C10 El sello & Cloelsello \\
\hline & C11 Hotel Terranostra & C11 Hotel Temranostra & C11 Hotel Terranostra \\
\hline & (12 Dir. de Desarollo Cultural & C12 Dir, de Desarsolio Cultural & C12 Dir, de Desarnollo Cultural \\
\hline & C13 Puno Cafe & C13 Puro calt & C13 Purocale \\
\hline & C14 Bar Restaurant Origgins & C14 Bar Restaurant OHiggins & C14 Bar Restaurant OHliggins \\
\hline & C15 Edificio Brasil & C15 Edfikio prasil & C15 Edikio Brasl \\
\hline & C 16 Ex Consejo Municipal & C16 Ex Consejo Murikipal & C16ExConsejo Municipal \\
\hline & C17 Edeficio Pedro Montt & C17 Edfficio Pedro Montt & C17 Edfikio Pedeo Monst \\
\hline & C18 Bar Resturant det Bario & C18 Bar Restaurant del Barrio & C18 Bar Restaurant del Barrio \\
\hline & C19 Edifikio Av, Angentina & C19 Edrkio Avv. Angentina & C19 Edfikio Av. Acgeentins \\
\hline & Cro El Valenciano & Cro El Valenciano & CroElivilenciano \\
\hline & Cil Bar Restaurant Babones & C21 Bar Restaurent Barbents & C21 Bar Restaurant Barbones \\
\hline & C2z loft de is Adwana & C22 loh de L Aduana & C22 loh de L Adfuna \\
\hline & C23 tdício Bawestrello & C23 Edficio Bavestrelo & C23 Edificio Bawesterelo \\
\hline \multirow{8}{*}{$\begin{array}{l}\text { Espadios } \\
\text { privados }\end{array}$} & Di Luceo Matilde Brandas & & \\
\hline & D2 Edrkio Yungay & & \\
\hline & D3 Pobladibn Zenteno & & \\
\hline & D4 Poblacibon Obera Unión & & \\
\hline & OS Palacio Polanco & & \\
\hline & E1 Nomada Eco Hostel & & \\
\hline & E2 Edificio Generd Cruz & & \\
\hline & E3 Club Suboficiales Armuda & & \\
\hline TOTAL & S1casos & 39 casos & 23 CASOS \\
\hline
\end{tabular}

\begin{tabular}{|c|c|}
\hline FAMILIAS & SELECCION CASOS DE ESTUDIO \\
\hline \multirow{4}{*}{$\begin{array}{l}\text { Edificio } \\
\text { continuo } \\
\text { Cima de cerro } \\
\text { con mirador }\end{array}$} & CUARTA ETAPA \\
\hline & Gelinternoso \\
\hline & C4 Hoted Cra Vander \\
\hline & $C 7 D n 39$ \\
\hline \multirow{7}{*}{$\begin{array}{l}\text { Edificio } \\
\text { continuo } \\
\text { Ple de cerro } \\
\text { con calle }\end{array}$} & CSCrá Cenvecera Altamira \\
\hline & C6 Bar Industrial \\
\hline & Cola bya \\
\hline & C10elsello \\
\hline & C11 Hoted Terranostra \\
\hline & C) Rur Rectaurant Batbones \\
\hline & C23 Edificio Buneutrello \\
\hline \multirow{12}{*}{$\begin{array}{l}\text { Edificio } \\
\text { continus } \\
\text { Plan con calle }\end{array}$} & CI Edficio Sotominyot \\
\hline & C8cmaplan \\
\hline & C12 Dir. de Desarodo Cultural \\
\hline & C13 puro Caté \\
\hline & C14 Bar Restaurant OFiggins \\
\hline & C15 Edificio Brasil \\
\hline & C16ExConsejo Municipal \\
\hline & C17 Edificio Pedro Montt \\
\hline & C18 Ear Restaurant del Bario \\
\hline & C19Edificio Av. Argentina \\
\hline & Crofl vilenciuno \\
\hline & Cnlott de ba Aduana \\
\hline
\end{tabular}

"Obras de rethabilitación en proceso, inmueble cerrado al publico.
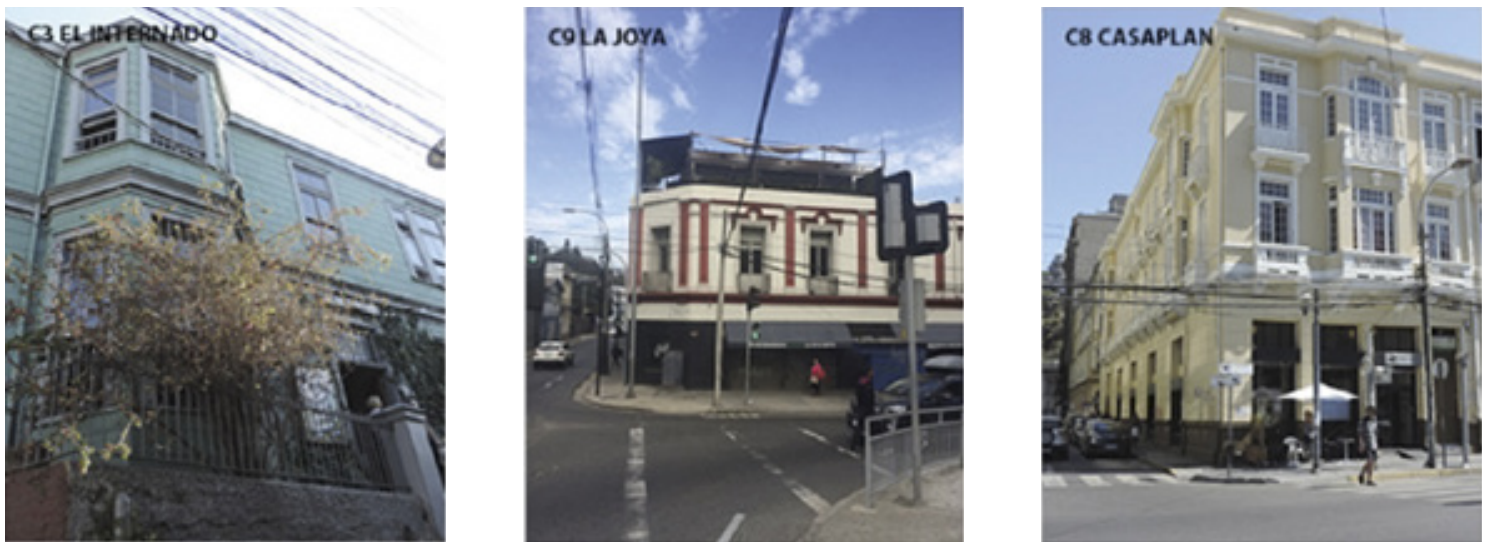

Tabla 1 Filtros en la selección de los casos de estudio. Fuente: Elaboración de Nicole Eujenio. 
diagnóstico de la microescala da cuenta de que existe un modo de intervenir que está ligado a las capacidades de lo construido que arroja oportunidades $y$, a la vez, delimita los posibles problemas. A esto se le ha denominado el "potencial de confort", que surge de la evaluación material, funcional y de comportamiento humano en un edificio preexistente y da como resultado un indicador que reconoce su capacidad para generar condiciones de confort ambiental, las que sirven para diseñar estrategias de rehabilitación. Los parámetros que determinan este potencial son la visión, la audición, la sensación y el olfato, asociados a las cualidades materiales, funcionales y de comportamiento evaluados a nivel de unidad, de bloque y de conjunto (Tabla 2). Estos antecedentes permiten crear un expediente base desde el cual nacen los lineamientos y el énfasis a considerar en la intervención, ya que indican las capacidades reales con las que cuenta el edificio. No obstante, resulta fundamental integrar el modo de vida de los habitantes y fomentar las buenas prácticas al interior de la vivienda que influyen directamente en el uso y desuso de los espacios.

La escala de valoración de las entrevistas permite reconocer y cuantificar de forma preliminar las variables de confort ambiental que tienden a la obsolescencia $y$, asimismo, las que tienden a reconocerse como un potencial de confort del edificio. Como se plantea en la introducción, en el caso de Valparaíso la vista y el asoleamiento se constituyen como bienes colectivos, lo que se refleja en el relato de los habitantes del conjunto cuando evalúan con una valorización de "bueno" o "excelente" respecto del confort visual y el acceso a las vistas que tienen las unidades. También sobresale la valoración positiva del "confort de sensación". Cabe destacar que las entrevistas se aplicaron durante el mes de julio, por ello se debe considerar las condiciones climáticas propias de la época, como los rangos de temperatura, las horas de luz día, la humedad ambiental, etc. Se entrevistó tanto a hombres como a mujeres de diversas edades, de los cuales el 50\% llevaba más de 20 años viviendo en el conjunto. En la otra mitad, había personas que llevaban entre 5 meses y 7 años de ocupación, por lo tanto, la idealización de los habitantes con el conjunto es igualmente relevante al momento de evaluar su vivienda y la agrupación.

Al reconocer el potencial de confort del edificio, a partir de la recomposición de las variables de confort ambiental identificadas y calificadas por el habitante, se logra caracterizar y medir la dimensión intermedia desde su contribución a las condiciones de confort ambiental, lográndose definir así un espesor de envolvente que debería ser materia central de la estrategia de rehabilitación sostenible.

\section{Meso escala: La capacidad de auto-regeneración socioespacial}

Comprender la obsolescencia de los edificios y brindarles una rehabilitación que oriente su futuro hacia la sostenibilidad requiere integrar la relación indisociable entre territorio y habitantes. Dicho vínculo es tangible en la dimensión intermedia de las viviendas colectivas, cuyo territorio se conforma por edificios, lugares, habitantes y territorios de mediación, acentuando el valor de lo común. Desde ese enfoque, cualquier actuación de rehabilitación debe brindar el espacio y las directrices para la actualización de lo construido y de las prácticas en el tiempo. A esta oportunidad se le ha denominado "capacidad de auto-regeneración socioespacial". Este concepto es la capacidad que poseen los habitantes para gestionar el territorio y actualizarlo de forma permanente, con el fin de responder a los cambios que requiere el hábitat construido. En concreto, este parámetro permite identificar las cualidades del espacio, la red de personas o entidades conectadas al territorio, sus capacidades para actuar en él y transformarlo según los requerimientos del colectivo. Reconociendo estas variables, es posible determinar el grado de intervención necesario a implementar, de modo que se visibilicen y potencien las capacidades particulares, y se integre el manejo de los ciclos de los habitantes hacia el desarrollo sostenible del hábitat construido, ya que visibilizar las prácticas que ocurren en el territorio e integrarlas en las actuaciones facilita la transformación social y conduce hacia la sostenibilidad (Calzada, Chautón y Di Siena, 2013). Esto se traduce en el aumento de la eficiencia de los territorios y de la preocupación por el bienestar común (Fariña, 2013). De allí, la información entregada por los habitantes se vuelve fundamental para detectar redes en el territorio no visibilizadas por la matriz de análisis y que decantan en un mapeo que articula la capacidad de auto-regeneración socio-espacial (Figura 5). Es importante señalar que la estructuración de la información da pie a determinar los diversos grados y factores de prioridad a considerar en la rehabilitación, revela también los posibles agentes/personas capaces de promover el territorio y liderar una comunidad más activa y compenetrada con la sociedad, e identifica, por último, a los posibles potenciadores y responsables de intervenir. En definitiva, se trata de un plan de trabajo y de gestión que, a través de actuaciones o estructuras morfológicamente simples, produce actividades fundamentales, es decir, una arquitectura como sistema que no posee una expresión morfológica definible, sino que emite diversos significados (Ito, 2000), logrando reactivar procesos en decadencia o fomentar activaciones de nuevas oportunidades de acción entre habitantes y agentes territoriales.

\section{Macroescala: La oportunidad de revitalización}

Cuando un edificio se encuentra en desuso, su entorno se ve directamente afectado y de manera desfavorable, ya que esta situación puede generar inseguridad, disminución de vida social a su alrededor o simplemente porque el lugar es evitado por los peatones. Una oportunidad para revertir esta obsolescencia es la rehabilitación arquitectónica, que puede abarcar una transformación formal, funcional o material, albergando y permeando la actividad hacia el espacio público. Pero ¿cuánto de esta rehabilitación efectivamente revitaliza el entorno brindándole una oportunidad al sector para la proliferación de nuevas actividades? Últimamente, el debate académico ha intentado relacionar la arquitectura y el urbanismo mediante la rehabilitación y se afirma que esta no debe actuar solo para los edificios, sino que debe incluir el entorno urbano (Yagüe, 2010). Se entiende que la rehabilitación debe concebirse 
VISIÓN

\begin{tabular}{|c|c|c|c|c|c|}
\hline \multicolumn{3}{|l|}{ VISIÓN } & \multicolumn{3}{|l|}{ AUDICIÓN } \\
\hline \multirow{19}{*}{ Material } & \multirow{2}{*}{ Fluje luminese } & Color de las superticies & \multirow{14}{*}{ Material } & \multirow{6}{*}{ Ruido aereo } & Percepción ruido exterior \\
\hline & & Color del entorno & & & \multirow{2}{*}{ Percepción del ruido de otres recintos } \\
\hline & \multirow{3}{*}{ Aluminandia } & \multirow{3}{*}{$\begin{array}{l}\text { Cantidad de luz sobre las superficies } \\
\text { de trabajo } \\
\text { Ubicacion de las superficies de } \\
\text { trabajo respecto a la fuente de luz }\end{array}$} & & & \\
\hline & & & & & $\begin{array}{l}\text { Percepción de ruldo de otras } \\
\text { unidades }\end{array}$ \\
\hline & & & & & Paso del sonido por grietsso \\
\hline & \multirow{2}{*}{ Luminancia } & Color de las superficies & & & hendiduras \\
\hline & & Color del entomo & & \multirow[b]{2}{*}{ Ruido impacto } & $\begin{array}{l}\text { Percepción de impactos de recintos } \\
\text { contiguos }\end{array}$ \\
\hline & \multirow{5}{*}{$\begin{array}{l}\text { Proporciones del } \\
\text { espacio }\end{array}$} & \multirow[t]{2}{*}{$\begin{array}{l}\text { Porcentaje de vanos en relación a las } \\
\text { superfices }\end{array}$} & & & $\begin{array}{l}\text { contiguos } \\
\text { Percepción de impactos de unidades } \\
\text { contiguas }\end{array}$ \\
\hline & & & & \multirow{2}{*}{$\begin{array}{l}\text { Ruido de } \\
\text { instalaciones }\end{array}$} & \multirow[t]{2}{*}{ Percepción de ruldo de instalsciones } \\
\hline & & Distribución de la iluminancia & & & \\
\hline & & \multirow{2}{*}{$\begin{array}{l}\text { Presendia de zonas oscuras en los } \\
\text { recintos }\end{array}$} & & \multirow[t]{2}{*}{ Caracteristicas } & Absorción de las superficies \\
\hline & & & & & Reflexión de las superficies \\
\hline & \multirow{7}{*}{ Orientación } & \multirow[t]{2}{*}{ Del bloque respecto al contexto } & & Propordiones & Relación ancho/alto/largo \\
\hline & & & & Orientación & Respecto al ruido de los recintos \\
\hline & & De la unidad respecto al contexto & \multirow{2}{*}{ Funcional } & \multirow{2}{*}{ Requerimientos } & \multirow{2}{*}{ Requerimientos de aislación de ruido } \\
\hline & & $\begin{array}{l}\text { Ingreso de luz respecto de la hora del } \\
\text { dia }\end{array}$ & & & \\
\hline & & De los recintos respecto a las vistas & \multirow{5}{*}{$\begin{array}{l}\text { De } \\
\text { comportamiento }\end{array}$} & \multirow{4}{*}{$\begin{array}{l}\text { Medidas de } \\
\text { protección }\end{array}$} & \multirow[t]{2}{*}{ Del ruido aereo } \\
\hline & & \multirow{2}{*}{ Del espacio comùn } & & & \\
\hline & & & & & Del ruido de impacto \\
\hline \multirow{3}{*}{ Funcional } & \multirow{2}{*}{ Orzanización } & Uso de los recintos & & & Del ruido de instalaciones \\
\hline & & Uso del espacio común & & Fuente de ruido & Respecto a la propla vivienda \\
\hline & Requerimientos & Requerimientos de iluminación & & & \\
\hline \multirow{3}{*}{$\begin{array}{l}\text { De } \\
\text { comportamiento }\end{array}$} & & Uso de iluminación artificial de aporo & & & \\
\hline & $\begin{array}{l}\text { Illuminadön } \\
\text { artificial }\end{array}$ & Uso de iluminación natural & & & \\
\hline & & Uso del espacio comùn & & & \\
\hline
\end{tabular}

\begin{tabular}{|c|c|c|c|c|c|}
\hline \multicolumn{3}{|l|}{ SENSACIÓN } & \multicolumn{3}{|l|}{ OLFATO } \\
\hline \multirow{14}{*}{ Material } & \multirow{4}{*}{ Disipación de calor } & Conel aire & \multirow{4}{*}{ Material } & \multirow[b]{2}{*}{ Flujo de aire } & \multirow{2}{*}{ Infiltraciones no controladas } \\
\hline & & Con th a & & & \\
\hline & & Con la humedad & & & Posición de las aberturas \\
\hline & & Con las superficies & & Aberturas & Relación entre aberturas \\
\hline & \multirow{2}{*}{$\begin{array}{l}\text { Temperatura } \\
\text { operativa }\end{array}$} & \multirow{2}{*}{$\begin{array}{l}\text { Relación entre temperatura del aire y } \\
\text { temperatura de las superficies }\end{array}$} & \multirow{3}{*}{ Función } & \multirow{3}{*}{ Contaminadión } & hadice de contaminación \\
\hline & & & & & Tiempo de cxupación de recintos \\
\hline & \multirow{2}{*}{$\begin{array}{l}\text { Distribucion de } \\
\text { temperaturas }\end{array}$} & \multirow{2}{*}{$\begin{array}{l}\text { Relación entre temperatura de las } \\
\text { superficies }\end{array}$} & & & Presencia de patogenos \\
\hline & & & \multirow{5}{*}{$\begin{array}{l}\text { De } \\
\text { comportamiento }\end{array}$} & Estrategias & De ventilación controlada \\
\hline & \multirow{6}{*}{ Orientación } & Respecto del sol de la unidad & & \multirow{4}{*}{ Contaminadión } & Por calefacción \\
\hline & & & & & Por humedad \\
\hline & & Respecto del sol de los recintos & & & \\
\hline & & & & & Por ollores \\
\hline & & Respecto a un foco de calor interno & & & \\
\hline & & Respecto del sol del espacio común & & & \\
\hline Función & Requerimientos & Rango de temperatura de bienestar & & & \\
\hline \multirow{5}{*}{$\begin{array}{l}\text { De } \\
\text { comportamiento }\end{array}$} & \multirow{3}{*}{ Prácticas } & Practica de calefación & & & \\
\hline & & Practica de enfriamiento & & & \\
\hline & & Prácticas de ventilación & & & \\
\hline & Vestimenta & Tipo y cantidad de vestimenta & & & \\
\hline & Actividad & Npo de actividad que realiza & & & \\
\hline
\end{tabular}




\section{PREPRINT}

AS54

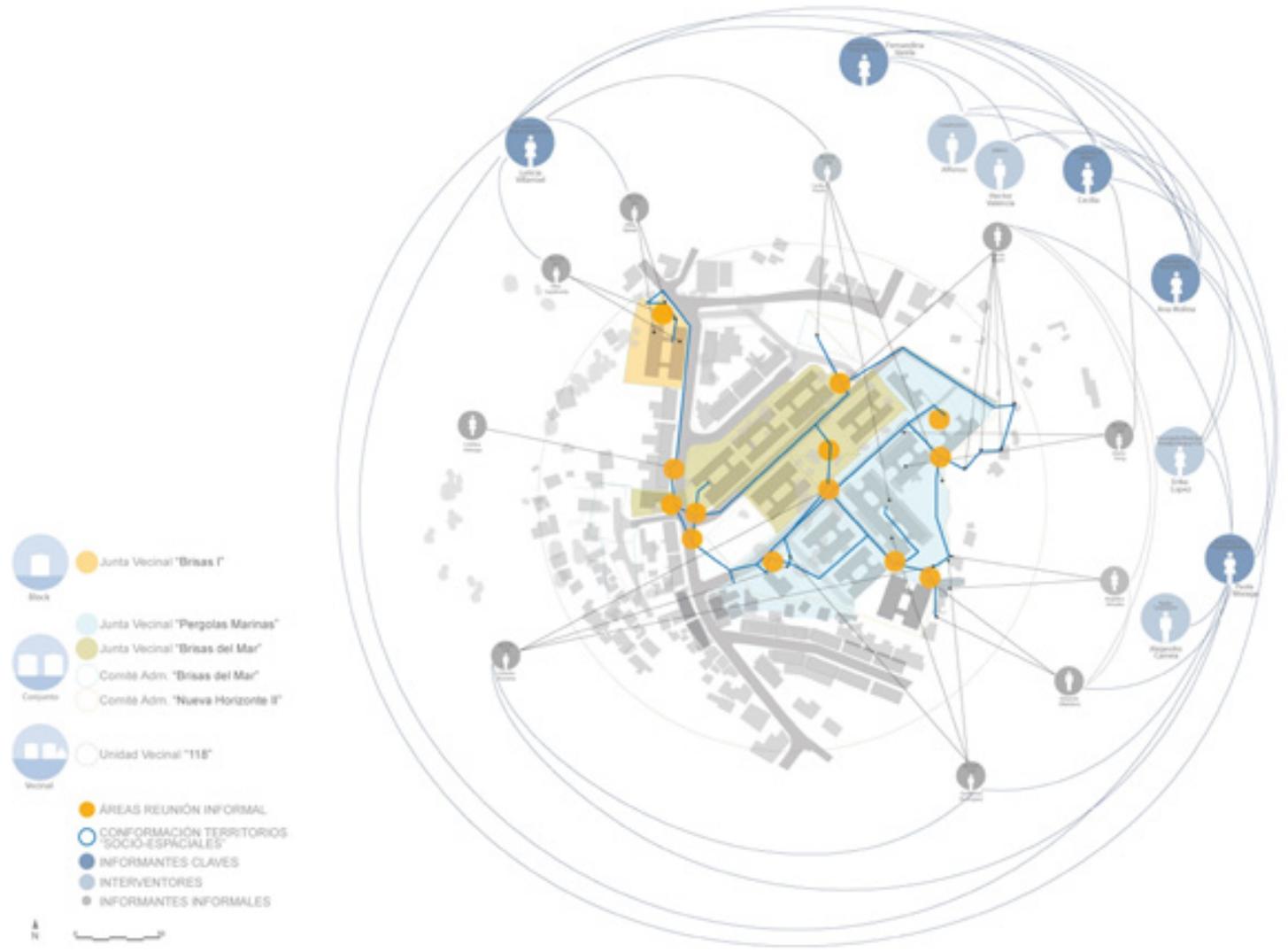

Figura 5 Mapeo de la "capacidad de auto-regeneración socioespacial”. Elaboración de Christian Alvial.

\begin{tabular}{|c|c|c|c|c|c|c|c|c|c|}
\hline & \multirow{2}{*}{\multicolumn{6}{|c|}{ REVITALIZACION }} & \multirow[b]{3}{*}{ Personalizacion } \\
\hline & & & & & & & & & \\
\hline & & & Lepbadad & 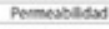 & Variedsod & Vensutiosod & Imagen apropads & Riquera perceptiva & \\
\hline \multirow{11}{*}{ 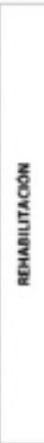 } & \multirow{3}{*}{$\frac{5}{8}$} & $\begin{array}{l}\text { Semipubtico } \\
\text { Semiprivedo }\end{array}$ & & & $\bullet$ & - & & & \\
\hline & & $\begin{array}{l}\text { Semiprivads } \\
\text { Privato }\end{array}$ & & & - & $\bullet$ & & & \\
\hline & & Semiprivadoscomplementaries & & & - & • & & & \\
\hline & \multirow{4}{*}{$\stackrel{\varrho}{5}$} & Conservadion & - & & & & - & & \\
\hline & & Adicion & - & & & - & - & & \\
\hline & & Sustraciobn & & - & & - & & & \\
\hline & & Adaptacion & & - & & & - & - & \\
\hline & \multirow{4}{*}{ है } & Envolvente & & & & & - & $\bullet$ & - \\
\hline & & Estruction & & & & & & & $\bullet$ \\
\hline & & Segunidiad & & & & & & & $\bullet$ \\
\hline & & Habenabildad & & & & & & & • \\
\hline \multicolumn{10}{|c|}{ OPCRTUNIDAO DE REVIRUUZACION } \\
\hline & \multicolumn{2}{|c|}{ Edeficio Aacensor } & \multicolumn{2}{|c|}{ Edfocho aldado } & Edaticle coms & nediuneros & Esticio consmuo engins & Eathoba & so doble espans \\
\hline & \multicolumn{2}{|c|}{$\begin{array}{l}\text { En cima y ple de cerro } \\
\text { Con minador ceremono }\end{array}$} & $\begin{array}{l}\text { En oma ypit } \\
\text { Con mirador }\end{array}$ & cesoso & \multicolumn{2}{|c|}{$\begin{array}{l}\text { Encimude certo } \\
\text { Con mirabor cerceno }\end{array}$} & $\begin{array}{l}\text { Enple decenso } \\
\text { Con vistas } x \text { ceems }\end{array}$ & \multicolumn{2}{|c|}{$\begin{array}{l}\text { Enei plan } \\
\text { Con alameda cercana }\end{array}$} \\
\hline
\end{tabular}

Tabla 3 Síntesis de la oportunidad de revitalización y sus relaciones con la rehabilitación. Fuente: Elaboración de Nicole Eujenio. 
desde lo arquitectónico y lo urbano, en su interacción, y no de manera diferenciada, ya que cada vez más el entorno es entendido como una prolongación del espacio privado habitado (Fariña, 2013). De esta manera, existen aspectos de la arquitectura que contribuyen a la vida pública del espacio urbano, o sea, a la revitalización. Este efecto ocurre tanto desde la preexistencia como desde la rehabilitación de estos espacios, agregando niveles de privacidad entre el ámbito público y el ámbito privado, y dando cabida a la dimensión intermedia, lugar donde se presenta la citada oportunidad de revitalización. Este concepto consiste en que tanto el edificio como el entorno pueden tener condiciones que potencien su uso y el desarrollo sostenible del lugar, pero no es sino hasta que se rehabilita que es posible visibilizar dicha potencialidad. La vitalidad de un entorno construido radica en la capacidad que tenga este de proveer a sus usuarios la posibilidad de elección (Bentley et al., 1999), en consecuencia, la revitalización entrega mayores posibilidades de utilizar los edificios y sus alrededores, para lo cual estos lugares deben ser más receptivos, atrayendo el encuentro social.

La oportunidad de revitalización está ligada con la calidad del diseño arquitectónico y éste se vuelve aún más relevante en contextos consolidados con un patrimonio arquitectónico sensible, como lo es Valparaíso. Además, se identifica que la tipología de edificio continuo es, en esta ciudad, el más recurrente y coincide con los espacios semiprivados (23 casos). Entonces, la oportunidad se manifiesta dentro de los aspectos variables presentes, tanto en el edificio como en su entorno, y en el grado de rehabilitación que se esté dispuesto a llevar a cabo. Por un lado, en contextos con revitalización preexistente mayor, las actuaciones de rehabilitación pueden abarcar menos aspectos revitalizantes, mientras que en contextos con algún grado de revitalización más baja, éste debe identificar qué actuaciones de rehabilitación son más beneficiosas en relación a los aspectos requeridos para la revitalización (Tabla 3).

Recientemente, en la ciudad-puerto se han efectuado variadas propuestas de rehabilitación arquitectónica. Los proyectos han sido estratégicos al momento de escoger la preexistencia. La mayoría de ellos actúan en edificios insertos en situaciones urbanas accesibles, que forman parte de una red de conectividad que los sitúa, a su vez, cerca de otros hitos importantes de la ciudad, potenciando su funcionamiento. Por otro lado, los proyectos de rehabilitación en la ciudad de Valparaíso mantienen una relación más bien visual con el espacio público. Las estrategias más representativas son las que generan un nuevo espacio intermedio, potenciando las relaciones con el entorno. También se pudo observar el aprovechamiento de los vanos para hacer visibles las actividades dentro y hacia el exterior. Sin embargo, extender las actividades hacia el exterior es un recurso que solo se hace parte como instalación temporal y no como una apropiación física de los proyectos (Figura 6). Con esto se demuestra que el límite externo es el encargado de albergar usos que sean beneficiosos para el entorno; aquí la planta baja y la fachada son los bordes capaces de contribuir con la vitalidad.

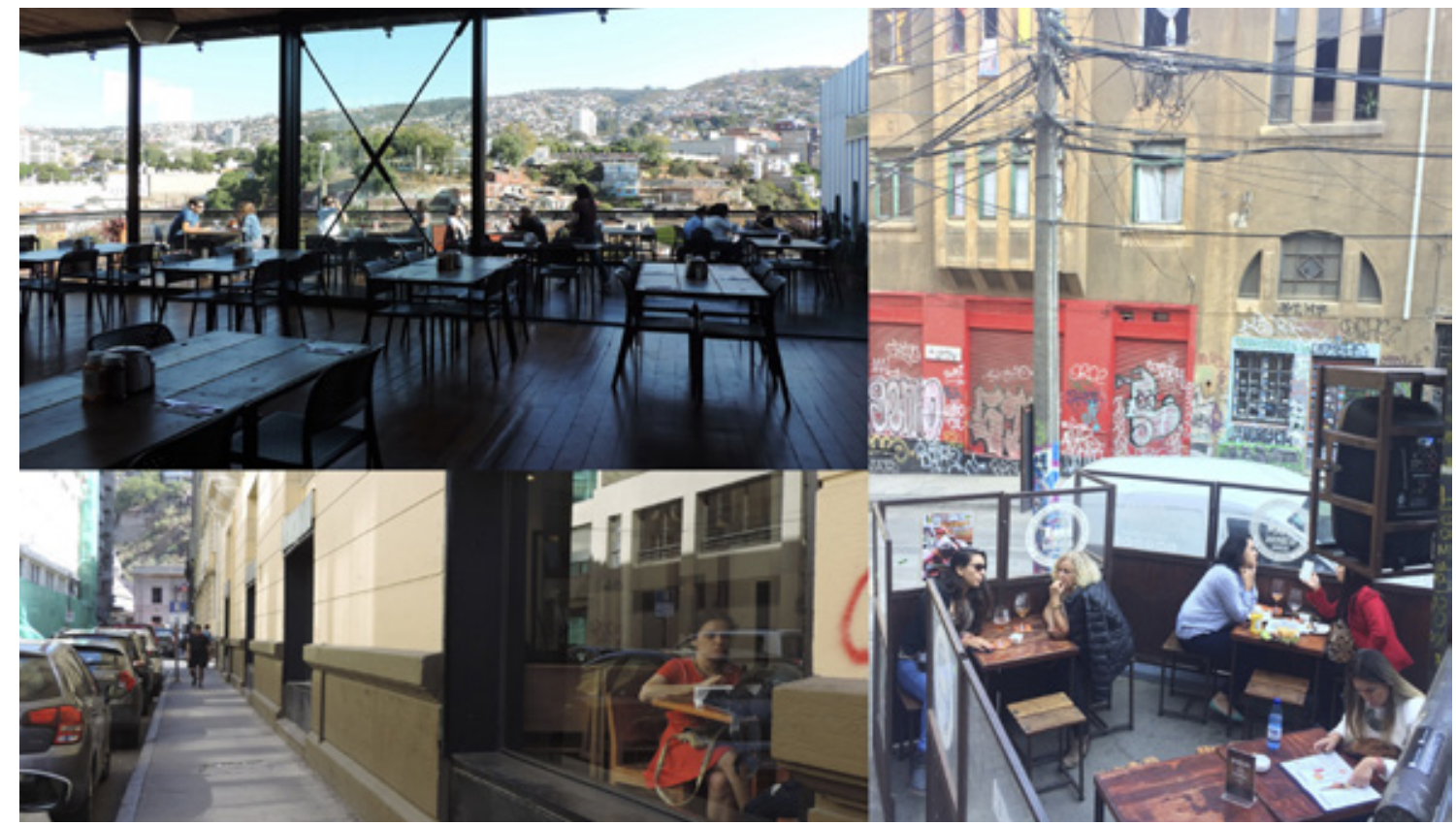

Figura 6 Relación visual con el espacio público y ocupación del entorno cercano. Fuente: Fotografia de Nicole Eujenio, marzo 2018. 


\section{CONCLUSIONES}

A escala de vivienda, la recomposición que se realiza de la totalidad de acuerdo a la relación que el habitante establece con el entorno construido, permite definir las características y límites del mismo, y así identificar cuáles son los atributos primitivos del edificio que condicionan las variables de confort ambiental. Estos son cualificados en términos de su tendencia al confort o a la obsolescencia, por medio de la percepción y significación del habitante, a partir de lo cual surge el "potencial de confort" como indicador del conjunto de características que propenden las condiciones ambientales al confort.

A escala de conjunto, una rehabilitación sostenible ha de entenderse medioambiental, social y financieramente, para dotar a la vivienda colectiva de un nuevo ciclo de vida que reconozca el equilibrio adecuado entre valores patrimoniales y la transformación necesaria de la arquitectura, ya que mantener vivo el patrimonio implica la transformación necesaria y funcional compatible con la memoria (Chacón, 2012). Además, debe entregar las herramientas necesarias para que se reconozca su "capacidad de auto-regeneración socioespacial" y responda ante las necesidades futuras de los habitantes reales y potenciales, desde un proceso que los haga partícipes de las decisiones y conscientes del ciclo de obsolescencia.

A escala urbana, las intervenciones deben ir más allá de la rehabilitación del edificio y contribuir al espacio urbano, incrementando la actividad del entorno y fomentando el encuentro social. En las rehabilitaciones de Valparaíso, la tendencia es al cambio funcional de los inmuebles, el que se orienta a servicios diversos vinculados al comercio y cultura, aportando con usos colectivos a los sectores involucrados. No obstante, la obsolescencia arquitectónica y urbana se minimiza en la medida que las intervenciones aporten con variedad y versatilidad de funciones, y destacarán aquellas que introduzcan contribuciones al entorno gracias a su estructura espacial o calidad arquitectónica, e integren interiorexterior de manera formal, especialmente cuando la preexistencia no lo manifiesta, para generar límites permeables que contribuyan con la vitalidad urbana.

Finalmente, cabe enfatizar que, para identificar una estrategia genérica de rehabilitación arquitectónica sostenible, el trabajo con tres escalas de análisis es pertinente y valioso, ya que cada una de ellas da cuenta de la complejidad presente en dicho territorio, lo que requiere de precisión respecto de los parámetros fundamentales que actúan en cada caso. Solo mediante esta definición base es posible integrar todas las escalas a una herramienta integrada de análisis que evalúe el mundo interno, el externo y el intermedio. En cuanto a las herramientas desarrolladas para las escalas micro y meso, se requiere de su aplicación en más casos de estudio para identificar variantes e invariantes; mientras que en la escala macro, se precisa de la integración de la visión del habitante para complementar el estudio morfológico. 
BAILLY, Antoine. Lo imaginario espacial y la geografía: En defensa de la geografía de las representaciones, Anales de Geografía, Universidad Complutense, 1989, vol. 9, pp. 11-19.

BENTLEY, Ian; ALCOCK, Alan; MCGLYNN, Sue; MURRAIN, Paul y SMITH, Graham. Entornos Vitales. Hacia un diseño urbano y arquitectónico más humano. Manual Práctico. $1^{\text {a }}$ ed. Barcelona: Editorial Gustavo Gili, 1999.

BUBER, Martin. ¿Qué es el hombre? México: Fondo de Cultura Económica, 1967.

CALDUCH, Juan. El declive de la arquitectura moderna: deterioro, obsolescencia, ruina, Palapa, 2009, vol. 4, pp. 29-43.

CALZADA, Igor; CHAUTÓN, Adolfo y DI SIENA, Domenico. \#MacroMesoMicro. Marco Sistémico de Territorio desde la Innovación Social [en línea]. Edición propia, 2013. [Consultado 20 noviembre 2018]. Disponible en: http:// macromesomicro.com

CARRASCO, Carolina. La espacialidad del encuentro. Análisis de las comunidades de los conjuntos habitacionales modernos en el caso chileno del Gran Valparaíso. Universidad Politécnica de Madrid, ETSAM, Departamento de Composición Arquitectónica, 2015.

CHACÓN, Eva. El reciclaje del hábitat social colectivo. Estrategias y tecnologías. Universidad de Granada, EscuelaTécnica Superior de Arquitectura, Departamento de Construcciones Arquitectónicas, 2012.

FARIÑA, José. Ciudad sostenible, rehabilitación arquitectónica y regeneración urbana, Monografías de la Revista Aragonesa de Administración Pública, 2013, vol. XV, pp. 15-26.

GEHL, Jan. La humanización del espacio urbano: la vida social entre los edificios. $1^{\text {a }}$ ed. Barcelona: Editorial Reverté, 2006.

GOLDBERGER, Paul. Porqué la arquitectura importa. Madrid: Ivorypress, 2012.

HAUSLADEN, Gerhard; SALDANHA, Michael; LIEDL, Petra y SAGER, Christina. Climate Design. Solutions for buildings that can do more with less technology. München: Birkhauser, 2004.

HERTZBERGER, Herman. Lessons for students in Architecture. Rotterdam: Editorial 010 Publishers, 1991.

ITO, Toyo. Escritos. Murcia: Colegio oficial de aparejadores y arquitectos técnicos, 2000.

KAPSTEIN, Glenda. Espacios Intermedios: Respuesta arquitectónica al medio ambiente. Santiago: ARQ Ediciones, 2015.

LUCCHI, Elena. Sostenibilidad y eficiencia energética de los edificios históricos, PH: Boletín del Instituto Andaluz del Patrimonio Histórico, 2015, vol. 23, n 88, pp. 230242.
MONTANER, Josep; MUXÍ, Zaida y FALAGÁN, David. Herramientas para habitar el presente. La vivienda del siglo XXI. Barcelona: Universitat Politécnica de Catalunya, 2011.

NORBERG-SCHULZ, Christian. Existencia, Espacio y Arquitectura. Barcelona: Editorial Blume, 1975.

TUAN, Yi Fu. Space and Place. The perspective of experience. Londres: Ed. Arnold, 1977.

VAN EYCK, Aldo. On inside and outside space. En: VAN EYCK, Aldo; LIGTELIJN, Vincent y STRAUVEN, Francis. Aldo van Eyck Writings: collected articles and other writings 1947-1998. Amsterdam: SUN, 2008, p. 288.

VAN EYCK, Aldo. A home for Twin Phenomena. En: VAN EYCK, Aldo; LIGTELIJN, Vincent y STRAUVEN, Francis. Aldo van Eyck Writings: collected articles and other writings 1947-1998. Amsterdam: SUN, 2008, p. 61.

YAGÜE, José María. Revitalización vs Rehabilitación [en línea]. Ponencia presentada en Congreso Internacional de Rehabilitación y Sostenibilidad. El futuro es posible. Barcelona, 2010. [Consultado 16 octubre 2018]. Disponible en: www.rsf2010.org/files/u1/A18.pdf 